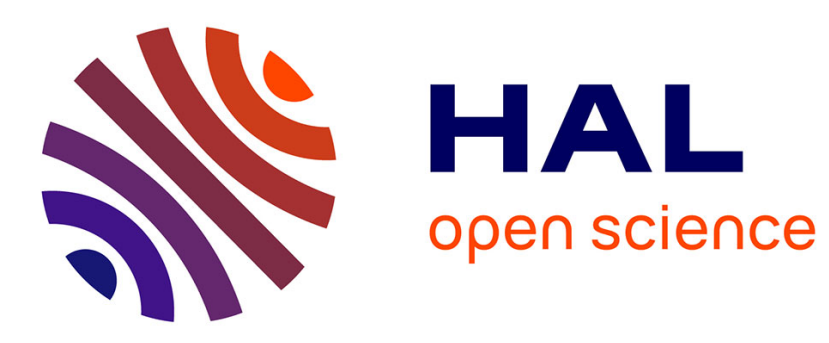

\title{
XaaS Multi-Cloud Marketplace Architecture Enacting the Industry 4.0 Concepts
}

\author{
Adrián Juan-Verdejo, Bholanathsingh Surajbali
}

\section{To cite this version:}

Adrián Juan-Verdejo, Bholanathsingh Surajbali. XaaS Multi-Cloud Marketplace Architecture Enacting the Industry 4.0 Concepts. 7th Doctoral Conference on Computing, Electrical and Industrial Systems (DoCEIS), Apr 2016, Costa de Caparica, Portugal. pp.11-23, 10.1007/978-3-319-31165-4_2 . hal-01438257

\section{HAL Id: hal-01438257 \\ https://hal.inria.fr/hal-01438257}

Submitted on 17 Jan 2017

HAL is a multi-disciplinary open access archive for the deposit and dissemination of scientific research documents, whether they are published or not. The documents may come from teaching and research institutions in France or abroad, or from public or private research centers.
L'archive ouverte pluridisciplinaire HAL, est destinée au dépôt et à la diffusion de documents scientifiques de niveau recherche, publiés ou non, émanant des établissements d'enseignement et de recherche français ou étrangers, des laboratoires publics ou privés. 


\title{
XaaS Multi-Cloud Marketplace Architecture Enacting the Industry 4.0 Concepts
}

\author{
Adrián Juan-Verdejo ${ }^{1,2}$, Bholanathsingh Surajbali ${ }^{3}$ \\ ${ }^{1}$ Smart Development Centre, CAS Software AG, Karlsruhe, Germany \\ ${ }^{2}$ Information Systems Chair, Stuttgart University, Germany \\ ${ }^{3}$ School of Communication and Computing, Lancaster University, UK \\ b.surajbali@comp.lancs.ac.uk
}

\begin{abstract}
Cloud computing in conjunction with recent advances in Cyber-Physical Systems (CPSs) unravels new opportunities for the European manufacturing industry for high value-added products that can quickly reach the market for sale. The Internet of Things joins the Internet of Services to enact the fourth industrial revolution that digitalises the manufacturing techniques and logistics while pushing forward the development of improved factories with machine-to-machine communication delivering massively customised products tailored to the individualised needs of the customer. Interconnected CPSs using internal and cross-organizational services cooperate in real time increase the business agility and flexibility of manufacturing companies. Using CPS with cloud computing architectures leverage data and services stored and run in cloud environment from different vendors that usually offer different service interfaces to share their services and data. However, when using such architectures data silos appear and different vendors having different service interfaces can easily result in vendor lock-in issues. This paper proposes a multi-cloud marketplace architecture leveraging the existing myriad of different cloud environments at different abstraction levels including the Infrastructure-, Platform-, and Software-as-a-Service cloud models-that is, Everything as a Service or XaaS - delivering services and with different properties that can control the computation happening in multiple cloud environments sharing resources with each other.
\end{abstract}

Keywords: Cloud Computing, Cyber-Physical Systems, Migration, XaaS, decision making, Industry 4.0, IoT.

\section{Introduction}

Rapid advancements in the embedded intelligence that mobile devices provide through the use of their wireless sensors in a connected world thanks to modern mobile communications have led to the development of CPSs. Interconnected systems and systems of systems deliver a myriad of applications in the environmental, aerospace, civilian, military, industry, and government sectors. Pervasive computing, sensing technologies, smart environments—-such as smart homes or smart grids-, and computer 
networks unveil new opportunities in the Cyber-Physical research and industrial worlds [1]. But the limited ability of mobile devices and pervasive systems to run computation-intensive jobs arise challenges because of their limited computing power and battery life. The cloud computing model deliver advanced CPSs to offer solutions to this specific challenges and pave the way for implementing the Industry 4.0 concepts [2]. Industry 4.0 includes the so-called Smart Factory with CPSs monitoring physical processes creating a virtual copy of the physical world to make effective and accurate decentralised decisions [3]. In the Internet of Things (IoT), interconnected CPSs cooperate in real time through internal and cross-organizational services to deliver value-added complex products and services [3]. Disruptive advances in software and hardware together with the cross-pollination of concepts and the integration of information and communication in industrial automation and control systems provide new architectures. These architectures incorporate current advances in industrial automation aiming at increasing the business agility and flexibility not to mention mass customisation [4].

The motivation to this research work is to facilitate the delivery of the cloud-based Cyber Physical Systems of tomorrow whereby the upcoming Industry 4.0 and the IoT can build on top of cloud computing systems with many resources storing, processing, and sharing analysed information in real time from anywhere. Regardless the huge potential the interplay of all these technologies offer, at the same time new questions related to the selection of which cloud environment to use and the appearance of the data silo problem due to the vendor lock-in. A repository of fixed data under the control of one organisation's entity in isolation from the rest of the organisation or data silo resembles the grain stored in a farm silo removed from outside elements [1]. For organisations, the decision of which cloud service model to choose for their applications or decision on how to change from one cloud service model to another are becoming increasingly complex due to the cloud offerings heterogeneity and affects the decision making and how to adapt the application so that organisations can migrate it.

This paper answers the research question of how to deliver a multi-cloud architecture across multiple layers of the cloud stack that on the one hand avoids the portability and vendor lock-in issues while unravelling the whole potential behind modern computing architectures for IoT deployments that enable enacting the Industry 4.0 concepts. The rest of paper is organised as follows. Section 2 provides the research motivation for CPS, followed by Section 3, provides a taxonomy of cloud offerings, followed by Section 4 our proposed XaaS Multi-Cloud Marketplace Architecture to address CPS challenges applied to the Industry 4.0. Next, in Section 5 presents a discussion of our contribution and the related literature. Finally, we offer our conclusion and future work in Section 6.

\section{Motivation for Cloud-enabled Cyber-Physical Systems}

Very different domains-from agriculture and meteorology to biomedicine and computer vision including emergency response [1], automation, and aerospace but many others 
too-use technologies as varied as sensor-actuator networks, machine-to-machine communication, supervisory control, data acquisition. In addition to the multitude of applications domains, the conjunction of the IoT, services that cloud computing architectures deliver, and Cyber Physical Systems have triggered the emergence of the so-called fourth industrial revolution described. The Industry 4.0 collective term considers recent studies that state that in 2020 around twenty-six "Things" communicating through broad-band technologies. The digitalisation of the manufacturing techniques and logistics are pushing forward the development of Smart Factories with machine-to-machine communication delivering massively customised products tailored to the individualised needs of the customer. CPSs such as intelligent machines, storage resources, and utilities cater for a smarter production in real time respecting the stated requirements while delivering a competitive price. The EU therefore funds research aiming at bringing back to Europe production plants now outsourced to cheap-labour countries. This trend will strengthen the European manufacturing industry to deliver value-added products using machinery and robots with enhanced capabilities to achieve higher levels of quality, occupancy rate, and faster processing times. Due to the use of software deployed into systems with big data analysis capabilities and real-time intelligent monitoring and decision-making processes.

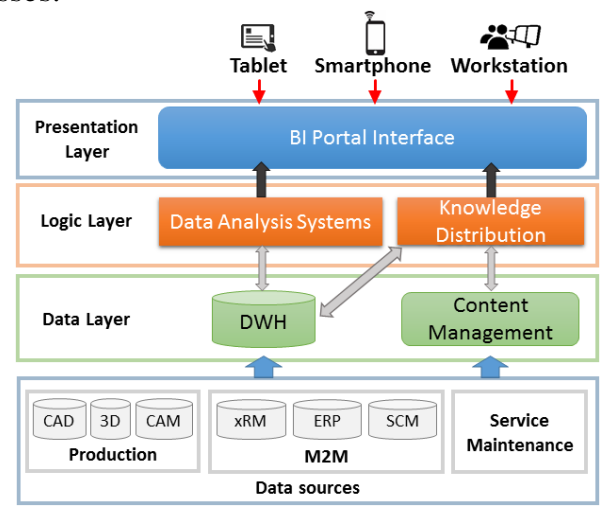

Fig.1. Generalised BI-enabled Industry 4.0 System (non-cloud-based)

New challenges related to Business Intelligence (BI) systems appear with the introduction of the Industry 4.0 [5]. Fast and accurate decision-making at strategic and operative levels paves the way to offering higher productivity, quality, and flexibility. Massive amounts of data coming from different sources-inside a company and across its boundaries-in different formats available in real-time must be analysed in real time to reach efficient solutions to problems and to improve the existing processes. This prompts the need for expertise from different areas including the manufacturing process, procurement, logistics, and product requirements. Given the large amount of data at hand and the time constraints; the analysis is best done in an automated manner based on the models of the existing knowledge domains and the data analysis processes to reach effective decisions. 
The integration of the Industry 4.0 and CPSs in a cloud-enabled environment delivers the needed connection through sensors and networks; computation, memory, and data on demand; advance modelling; content and context analysis and correlation; sharing and collaboration; and customisation. Cloud computing architectures facilitate the development of products using (micro) services delivered to consumers so that they configure products and services while reconfiguring manufacturing systems. Industry 4.0, cloud-based design and manufacturing, and cloud manufacturing adapt the cloud computing paradigm to the realm of computer-aided product development and the IoT. Error! Reference source not found. exemplifies the complexity of a BI-enabled Industry 4.0 systems whereby this paper shows the difficulty in selecting a multiple cloud offerings and adapting the system to use them in typical BI three-tier architectures. The data layer stores the structured and unstructured data coming from operational systems; the logic layer performs different analysis on those data; and the access layer let BI users access the high-level data they need [1]. Increasing the portability of such a system in a multi-cloud environment works under different interdependent constraints and success criteria specific to each organisation porting their system. Different dimensions that are not always equally important to all organisations and they can trade one for another one.

\section{Research Contribution to the Taxonomy of Current Cloud Offerings}

Nowadays organisations are increasingly moving the computing infrastructure supporting their manufacturing and enacting the entire supply chain from the traditional on-premises approach to current cloud computing environments. That way, ubiquitous networking service management and application opens the way to the reuse of services, cost reduction, flexibility, and increasing collaboration opportunities. Migrating to cloud deployments and using cloud-based services generate additional value due to the amount of data coming from different data sources. However, the data silo problem and vendor lock-in issues arise creating a gap of service interface and data model interoperability. The data of customers risk staying in individual data silos. At the same time organisations want to benefit from the characteristics of cloud environments but worry about the deployment efficiency or level of security the cloud provider achieves. Additionally, organisations may face the vendor lock-in problem when they want to migrate their system from one cloud provider to another one. The vendor lock-in risk stems from the lack of cloud vendors compliance to standards which makes the portability of data, application, and infrastructure difficult. But even if portability is supported in theory, the high complexity and the switching costs incurred still discourage organisations from porting their applications [6]. Hence, organisations developing applications should be able to choose between different cloud offerings by selecting the most reliable, well-reputed, or cost-efficient offering; or simply the one that meets their technical or business requirements. Organisations should also be able to switch easily and transparently 
between cloud providers whenever needed-for example, due to an SLA breach or when the cost is too high-without setting data and applications at risk-for example, loss of data or inability to run the application on a different platform. Moreover, they should be able to compare cloud offerings with different characteristics—such as resource, pricing, or quality of service-and to choose the one that best matches the computing needs of their services and applications [7]. A number of different cloud providers exist today making it difficult to select the most adequate cloud environment for an organisation. Organisations choose whether to move their applications to a different cloud service model or provider according to their current and future needs and how to do it. The cloud offering taxonomy the authors propose permeates across the whole architecture of the XaaS Multi-Cloud Marketplace and the Multi-Cloud Portability Tier and Broker. Fig. 2 shows the Cloud Offering Decision Making Module that selects a cloud offering configuration to use for a particular application according to the dimensions an organisation selects as important of each of the cloud deployment models from IaaS up to SaaS.

IaaS Offerings: IaaS resides at the bottom of the cloud service model and the rest of levels in the stack build on top of IaaS. Providers offer their resources, both physical and virtual: load balancers, virtual machines, servers, storage, and network. Given the particularities of the IaaS level, literature study, and the BI use case presented; this paper describes six dimensions to analyse different IaaS offerings as shown in Fig. 2. The IaaS Service Characteristics Dimension: Each VM instance type offers different price, number of virtual cores, available memory, and bandwidth for input and output traffic. The IaaS service characteristics include descriptions related to network access and service configuration. Additionally, IaaS let their consumers deploy their systems according to the private, community, public, or hybrid cloud deployment model. The Hardware Infrastructure Dimension: IaaS providers define their hardware infrastructure in terms of processing and memory. They define the types of server, processor (CPU), and memory, as well as the instance capacity. The Network Infrastructure Dimension: Systems deployed on IaaS environments share the provider's network resources with different network connection bandwidth and network infrastructure. The Repository Infrastructure Dimension: IaaS providers supply different database storage and might offer virtual private cloud possibilities. The tendency in regard with messaging services is to commoditise this service. An example is the Amazon Simple Queue Service (SQS) which liberates their consumers from maintaining their own service built by using a messaging implementations such as the Java Message Service (JMS) and the Microsoft Message Queuing. The Infrastructure SLA Dimension: The contractual agreement on the level of service to be provided by the IaaS service provider to the IaaS cloud consumer specifies numerous service performance metrics and their corresponding service-level objectives. Infrastructure SLA includes agreements related to the resource provisioning; and the service availability, reliability, security, geographical location, and elasticity. The SLA also specifies the service and auditing, disaster management, energy consumption, and the cost. The infrastructure cost includes defining the price level, as well as the billing metrics. The IaaS Portability Standardisation Dimension: Standardisation minimises the 
cost of porting software already developed for an IaaS environment to a new platform. Respecting portability standards and following portability methodologies increases portability and avoids vendor lock-in at the IaaS level. IaaS cloud consumers need to port the software runtime environments including configurations and APIs. Typically, this involves VMs. The Distributed Management Task Force (DMTF) proposed the Open Virtualization Format (OVF) which is often used in IaaS. OVF provides a format for open packaging and distribution which outlines how to deploy, manage, and run a virtual appliance on a VM.

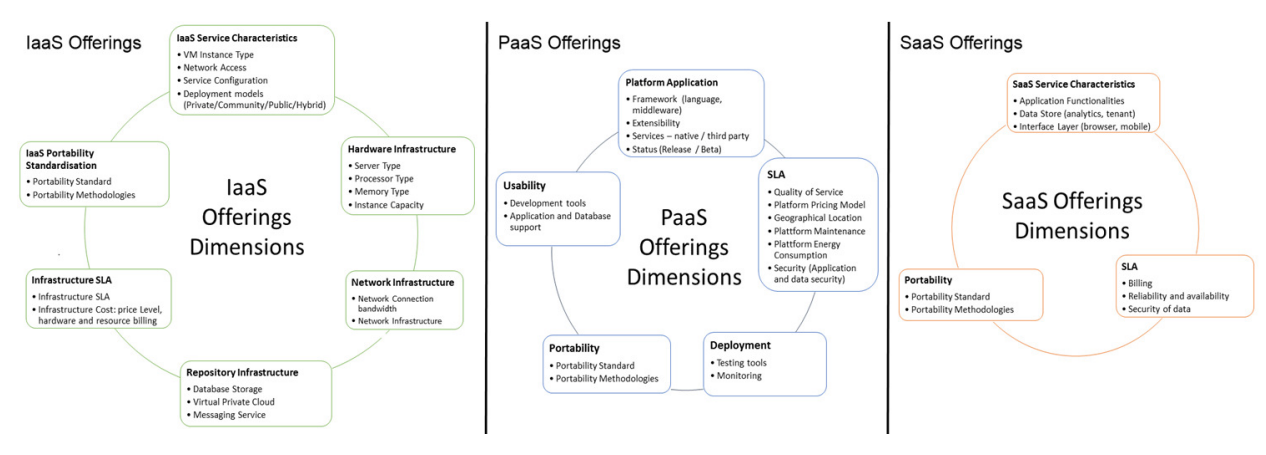

Fig. 2. IaaS, PaaS, and SaaS Offerings Dimensions

PaaS offerings: Fig. 2 shows the six PaaS offerings dimensions that come out from the analysis of the use case scenario described in Section 2 and differentiate PaaS offerings from one another. The Application Dimension consists of four sub-dimensions tightly related to the application whereas the SLA Dimension specifies the service contract in terms of quality of service, pricing model, geographical coverage, and availability. PaaS providers usually charge differently according to resources usage with billing that is fixed, metered, or hybrid [8]. Geographical coverage presents legal concerns such as those posed to EU-based companies that are not allowed to transfer any of their customer-related data outside of the European Union [9]. The Deployment Dimension describes how provider tools to validate and test the deployment of application and data components, from modeldriven approaches [10] to organisations requirements validation tools [1,7], and software development life-cycle tooling facilities. The data layer represents the asset of the BI user organisation interested on porting the BI system to PaaS environments. The portability of this layer to PaaS environments poses important security concerns for the organisation related to the trustworthiness of the PaaS provider in charge of storing the data layer and relates to the portability dimension. The Portability Dimension considers PaaS offerings providing information on the different standards they comply to in order to ensure application and data portability. The portability process itself has to keep data integrity and let users access them effectively. For example some users work with the data from different geographical locations. The authors consider three BI portability models for the 
private, public and hybrid PaaS cloud models. The private PaaS model whereby the organisation wants to ensure total data ownership through self-data security enforcement. The public PaaS model whereby the organisation trust cloud provider offerings. The hybrid PaaS model whereby the system can use more computational resources on demand while keeping sensitive data in the organisation premises. The Usability Offering Dimension considers the set of rapid PaaS application development tools that PaaS providers offer with different levels of support. Furthermore, PaaS offerings application and database usability varies as well as the training and documentation they offer. The usability dimension determines the application and database knowledge needed to maintain and extend an application deployed to a PaaS offering.

SaaS Offerings: Finally, the most abstract of the three fundamental cloud service models provides less degrees of freedom for the SaaS cloud consumer. As a result, fewer dimensions drive the migration decision to SaaS. The most important factor is the data migration as the solutions on SaaS are at the level of services: Customer Relationship Management (CRM), e-mail, enterprise resource planning (ERP), virtual desktop, management information systems (MIS), communication, games, computer-aided design (CAD) software. This paper proposes three dimensions within the SaaS level as shown in Fig. 2. The SaaS Service Characteristics Dimension: At this higher level of abstraction within the cloud computing service stack, the service characteristic dimension only involves the application functionalities, data store, and interface layer. The latter defines how the user of SaaS applications will access the service, such as browser or mobile interfaces. The SLA Dimension: SaaS providers formally define their services as a part of the service contract. The contractual agreement on the SaaS service level defines billing, reliability and availability, and security of the data. The Portability Dimension to enable applications to achieve SaaS portability by respecting portability standards and methodologies. SaaS portability can help them deal with cost increases, business stability issues, or SLA breaks by switching to a different SaaS provider. Data portability enables the use of data components across different applications.

\section{Proposed XaaS Multi-Cloud Marketplace Architecture}

The XaaS Multi-Cloud Marketplace Architecture in Fig. 3-as an architecture evolving from previous research works the authors did [7]—consists of three basic components including the XaaS Multi-Cloud Marketplace Broker, the XaaS Multi-Cloud Decision Support Tier and the XaaS Multi-Cloud Portability Tier. The XaaS Multi-Cloud Marketplace Broker allows end users to track IaaS, PaaS, and SaaS offerings, services, interdependencies, and offers through a marketplace. The main goal of the decision support broker is to make it easier, safer, and more productive for cloud adopters to navigate, integrate, consume, extend, and maintain cloud services through a marketplace. In this respect, the broker offers a range of value-added services to both cloud vendors and consumers, allowing organisations to lookup cloud offerings matching their 
requirements. Deploy the business logic of their application on one cloud offering and the underlying data on another without using additional software, changing application settings, or putting the application and data at risk. Efficiently select an alternative cloud offering and port the application to the new cloud offering with little development and decision effort. Transparently exchange data and application components with other cloud providers.

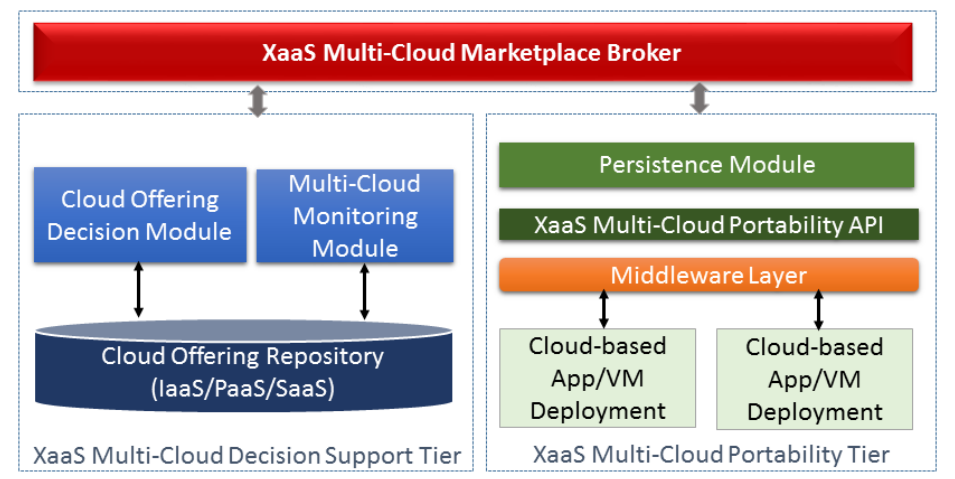

Fig. 3. Proposed XaaS Multi-Cloud Marketplace Architecture

The XaaS Multi-Cloud Decision Support Tier: this tier is responsible of the cloud offering selection and assessments taking into account the cloud offerings taxonomy from Section 3 above. The Cloud Decision Support contains the modules, which maintain the Cloud Offering Repository consistent and synchronised with the current offerings available. The Cloud Offering Decision Module supports seamless interaction between the cloud consumer and the cloud offering. Cloud consumers find available cloud offerings, which comply with their application's requirements and their criteria for porting their application. Additionally, the broker supports the application porting to cloud environments. This module implements the core functionalities such as cloud offering discovery, cloud offering analysis, cloud offering recommendation, and the InCLOUDer cloud offering selection and migration support from our previous work [1]. InCLOUDer addresses the multi-dimensional decision problem with the presented structured hierarchy of migration criteria. The hierarchy of criteria makes it easier for organisations to weight different criterion to one another and also complies with the natural nature of the migration criteria [7]. The cloud offering decision module facilitates the rating of a particular cloud offering by a SME's Software Engineer. Each engineer can leave a comment and rate a particular cloud provider, thus offering expressing their satisfaction or dissatisfaction with regards to quality, usability, reliability, and user-friendliness of the offering. The Multi-Cloud Monitoring Module monitors the deployed application and the cloud environment and manages the application's life-cycle. The Cloud Offering Repository includes the Unified Cloud API and the Cloud Offering Repository interacting 
with heterogeneous cloud offerings. It holds information on the capabilities, requirements, services, and restrictions of the cloud services across the three cloud service models. Once the cloud decision support tier recommends a cloud offering, the XaaS Multi-Cloud Portability Tier helps with the application deployment and porting from one cloud vendor to another one. Several models cater for deployment and porting process support. The Persistence Module handles the application dependencies that affect the processes to deploy, undeploy, start, stop, and port the target application to the target cloud provider. The dependencies range from operating system to executable formats, including compilers and libraries. The persistence module includes all these dependencies in the migration and allows developers to add more themselves. The XaaS Multi-Cloud Portability API supports the management of the deployed applications independently of the specific API of the underlying cloud offering. The Middleware is an adaptor between the cloud portability module and the native API of the cloud offering. More specifically, the middleware module translates the functions of the cloud Portability API to the native API of the cloud offering, and vice versa. As a result, the middleware module, allows the seamless communication among cloud offerings addressing the vendor lock-in concern.

The proposed XaaS Multi-Cloud Decision Marketplace Architecture addresses several challenges that Industry 4.0 practices. The architecture provides the necessary infrastructure catering for the end-to-end digital integration along the entire value chain. The cloud deployment provides advanced communication mechanisms within a virtualised infrastructure that makes smart factories oblivious to which factory executes which processes. The proposed architecture aims at allowing for the vertical integration that paves the way to achieving the high flexibility needed to support the zero (or near zero) lot size. The resulting cloud infrastructure allows for integrating assets such as sensors, actuators, mechanical parts, documents, or Intellectual Property with the interfaces between the "real world" and its IT representation and it continues with its communication towards the information layer to finally move to the functional layer that is the gate to the achievement of business goals. The architecture uses standards to allow worldwide value chains exchanging information for transfer of data and utilisation facilitating the horizontal integration from products to the connected world and the needed Collaborative Networks. Network partners can access competences of one another and respond to market needs in a sustainable manner. The proposed broker allows the access to collaboration spaces whereby companies have well defined access and writing when collaborating and where not so as to increase the levels of trust by offering control over the information companies exchange with other companies with which they compete. The portable cloud-based deployments allow for real-time monitoring of large amounts of sensor data, including RFID or similar technologies, about product data, the status and position of goods, and the status of the different components of a CPS that the architecture can seamlessly port to different environments to overcome the vendor lock-in and data silo problems. The resulting deployment connects the value chain across the entire product life cycle and allows for the agility needed for massive customisation. 


\section{Discussion and Related Literature}

The current evolution of general computation for individuals transforming into increasingly advanced pervasive devices has been accompanied with computing infrastructures moving to cloud computing environments. In conjunction with the trend towards miniaturisation, ubiquitous computing is a reality that the modern industry puts into play to improve and automate manufacturing, transport, and logistics. The interplay of technologies in the industrial domain ushers in the convergence of the physical and virtual world in the CPS allowing for the delivery of the infrastructure and ubiquitous computing fundamental to the fourth industrial revolution. Smart objects networked with the relatively new IPv6 addresses aggregate the information into modern cloud environments hosting advanced analytic (micro)services enhancing the decision making and control. As an example, manufacturers can timely react to changes such as in the supplier chain suddenly during production. Industry 4.0 opens the way for smoother changes in this network with manufacturers anticipating distortions in the supply chain by simulating, evaluating and selecting the best supply alternative. Organisations adopting the concepts behind the Industry 4.0 concepts have very special requirements tailored to their highly complex systems and can profit from the selection of particular cloud offerings at different levels of the cloud deployment stack. Additionally, organisations can minimise the data silo problem and portability issues with the use of multi cloud environments. In that respect, this paper proposes a multi cloud architecture with the use of a decision broker to schedule and deploy components to the appropriate XaaS cloud environment as well as services and (micro)services offered across the whole cloud service stack. Another contribution of our research work concerns the decision process involved in migrating to a cloud-enabled deployment by providing the basic mechanisms to help organisations find the best cloud provider offerings through a marketplace and mechanisms for cloud environment selection using the taxonomy of cloud offerings.

XaaS encompasses cloud-related services-from the Infrastructure- to the Software-as-a-Service cloud model including the Platform-as-a-Service model [11]running different cloud service models according to the famous NIST definition [12]. Concepts coming from cloud computing such as broad network access and resource pooling and from Service-Oriented Infrastructures (SOI) such as on-demand business-driven infrastructure and service-orientation [13] come together in XaaS. The selection of the best cloud model matching the current and future organisations' needs are becoming increasingly complex due to the number of cloud offerings to consider [1]. The proposed decision broker builds on top of the Analytic Hierarchy Process allowing formalising the cloud migration problem by defining the cloud migration criteria, the cloud service offerings, and the architecture and properties of the application. It is a multi-dimensional decision problem affected by a lot of factors, sub-factors, and parameters $[14,15]$ and our research work automate the process differing from some works [16, 17] improving the usage for real-case scenarios. The decision broker weights these alternatives by taking into account criterion metrics [1] and their importance relative 
to other criterion. When no metric is provided the weighting is done manually by the organisation based on their requirement needs. The migration of existing applications to cloud environments demands from organisations to select a suitable target cloud service $[17,18]$ and adapt their applications to them according to their needs and the specifics of their application [15]. Organisations have to evaluate many conflicting criteria before making any decision and the problem becomes a multiple criteria decision making problem [19]. Cloud4SOA differs from the proposed approach in that it requires the annotation of each provider offerings [20], and the annotation process of each offering is only feasible if there are established benchmarks of PaaS offerings so that all PaaS vendor offerings can be taken into consideration. The mOSAIC project [21] focuses on application portability at both IaaS and PaaS using a Cloud ontology to analyse the offerings of the cloud and using a brokering mechanism to search offerings requirements. Numerous Cloud API standardisation try to unify incompatible APIs coming from cloud providers with respect to cloud standards such as OCCI [22], TOSCA [23] and CIMI [24]; to PaaS standards such as the OASIS CAMP [25] which help avoiding vendor lockin. However, the adherence of a cloud standard at one cloud model for example at IaaS using OCCI with another provider adherence to another cloud at the PaaS using CAMP results in a standard incompatibility. To address standard incompatibility in portability across cloud models, we envisage extending our middleware layer in the cloud broker architecture in Fig. 3 to support cross-standard to interoperate with each other inspired by the research work from [26].

\section{Conclusion and Future Work}

Organisations adopting the concepts behind the Industry 4.0 concepts have very special requirements tailored to their highly complex systems and can profit from the selection of particular cloud offerings at different levels of the cloud deployment stack. We have presented a generic architecture-based approach to provide the necessary end-to-end digital integration along the entire value chain of cloud infrastructures. The information exchange for data transfer and utilisation facilitates the horizontal integration via standards to connect everything from products to the connected world in current worldwide value chains. In Collaborative Networks, network partners can access competences of one another and sustainably react to the market needs. Our broker is the door to collaboration spaces allowing organisations collaborate but keeping high levels of trust. This trust stems from the secured and controlled access of the information organisations exchange with one another including competing entities. The cloud-enabled advanced communication mechanisms connects smart factories executing different processes. In addition to horizontal integration, the architecture facilitates the vertical integration needed to provide high flexibility to adjust the production and lot size. The proposed cloud-based infrastructure integrates assets with their interfaces with its IT representation allows the communication and moving knowledge up to the information 
layer to finally from the functional layer achieve the organisations business goals. Using our approach and our XaaS Multi-Cloud Marketplace Broker organisations can minimise the data silo problem and portability issues with the use of multi-cloud environments and thereby addressing the vendor lock-in issue. Additionally, our proposed architecture eases the decision process using the author InCLOUDer framework to migrate cloud-enabled deployment from one cloud environment to another and provides the basic mechanisms to help organisations find the best cloud provider offerings through a marketplace. This is achieved using the cloud offering taxonomy that facilitates the selection of cloud offerings (IaaS, PaaS and SaaS) based on a number of offerings classification criteria with minimal effort.

As future work the authors will firstly face the validation phase with the quantitative and qualitative analysis of the ported cloud-enabled CPS infrastructure respecting the Industry 4.0 practices in terms of the cloud offerings dimensions relevant to the Smart Factory organisation and the end-to-end integration. Additionally we will confront the security and trust dimensions within the appropriate hierarchy in the cloud deployment model with the levels of trust and security at the level of Collaborative Networks. In a further phase, we will address the challenges with respect to standards incompatibility by building an interoperability middleware framework across cloud models where applications differences are seen in terms of incompatible interface signatures and data content and communication protocols across standardised layers of the cloud. The work will support the marketplace presented here with additional portable services tailored to the Industry 4.0. Services that will pool the monitoring information related to complex products and services to optimise the smart factory with regard to the logistics and manufacturing automation so as to seamlessly integrate with other business management applications. Organisations making use of the market place will have access to re-usable (micro)services supporting the addictive and subtractive manufacturing and to a myriad of off-the-self 3D designs ready to use.

\section{Acknowledgements}

The European Commission supported this work through the OSMOSE project FP7 610905 and the German Ministry of Education and research, BMBF, through the SysPlace Joint Project 01IS14018D. 
XaaS Multi-Cloud Marketplace Architecture Enacting the Industry 4.0 Concepts 23

\section{References}

1. Al Ali, R.a.G., Ilias and Gonzalez-Herrera, Inti and Juan-Verdejo, Adrian and Kit, Michal and Surajbali, Bholanathsingh. Towards a Framework for Compute-Intensive Pervasive Systems in Dynamic Environments. in 2nd International Workshop on Hot Topics in Cloud service Scalability. 2014. ACM.

2. Kagermann, H., et al., Recommendations for Implementing the Strategic Initiative INDUSTRIE 4.0: Securing the Future of German Manufacturing Industry; Final Report of the Industrie 4.0 Working Group. 2013: Forschungsunion.

3. Hermann, M., T. Pentek, and B. Otto, Design Principles for Industrie 4.0 Scenarios: A Literature Review. 2015.

4. Brettel, M., et al., How virtualization, decentralization and network building change the manufacturing landscape: An Industry 4.0 Perspective. International Journal of Science, Engineering and Technology 8 (1), 37, 2014. 44.

5. Givehchi, O.a.T., Henning and Jasperneite, Jurgen. Cloud computing for industrial automation systems-A comprehensive overview. in 18th Conference on Emerging Technologies \& Factory Automation (ETFA). 2013. IEEE.

6. McKendrick, J. Does Platform as a Service have interoperability issues. Cloud Commons, 2010.

7. Juan-Verdejo, A., et al. InCLOUDer: A Formalised Decision Support Modelling Approach to Migrate Applications to Cloud Environments. in 2014 40th EUROMICRO Conference on SEAA. 2014. Verona, Italy.

8. Kolb, S. and G. Wirtz. Towards Application Portability in Platform as a Service. in Proceedings of the 8th IEEE International Symposium on Service-Oriented System Engineering (SOSE). 2014.

9. Strobl, J., E. Cave, and T. Walley, Data protection legislation: interpretation and barriers to research. BMJ: Journal, 2000.

10. Fleurey, F., J. Steel, and B. Baudry. Validation in model-driven engineering: testing model transformations. in Model, Design and Validation, 2004. Proceedings. 2004 First International Workshop on. 2004. IEEE.

11. Duan, Y., et al. Everything as a Service (XaaS) on the Cloud: Origins, Current and Future Trends. in Cloud 2015. IEEE.

12. Mell, P. and T. Grance, The NIST definition of cloud computing. 2011.

13. The-Open-Group SOCCI Framework Technical Standard : Service-Orientation and Cloud Synergies. 2014.

14. Armbrust, M., et al., A view of cloud computing. Communications of the ACM, 2010. 53(4): p. 50-58.

15. Andrikopoulos, V., et al., How to adapt applications for the Cloud environment. Computing, 2013. 95(6): p. 493-535.

16. CSMIC Introducing the Service Measurement Index. Cloud Commons, 2011.

17. Garg, S.K., S. Versteeg, and R. Buyya. Smicloud: A framework for comparing and ranking cloud services. in Utility and Cloud Computing (UCC), 2011 Fourth IEEE International Conference on. 2011. IEEE.

18. Tran, V.X., H. Tsuji, and R. Masuda, A new QoS ontology and its QoS-based ranking algorithm for Web services. Simulation Modelling Practice and Theory, 2009. 17(8): p. 1378-1398.

19. Zeleny, M. and J.L. Cochrane, Multiple criteria decision making. Vol. 25. 1982: McGraw-Hill New York. 
24 A. Verdejo and B. Surajbali

20. D'Andria, F., et al. Cloud4soa: multi-cloud application management across paas offerings. in Symbolic and Numeric Algorithms for Scientific Computing (SYNASC), 2012 14th International Symposium on. 2012. IEEE.

21. Petcu, D., et al., Experiences in building a mOSAIC of clouds. Journal of Cloud Computing, 2013. 2(1): p. 1-22.

22. Edmonds, A. and T. Metsch. Open Cloud Computing Interface-Infrastructure. in Standards Track, no. GFD-R in The Open Grid Forum Document Series, Open Cloud Computing Interface (OCCI) Working Group, Muncie (IN). 2010.

23. Binz, T., et al., OpenTOSCA-a runtime for TOSCA-based cloud applications, in ServiceOriented Computing.2013, Springer.

24. Initiative, C.M. Cloud Infrastructure Management Interface. 2014.

25. OASIS Cloud Application Management for Platforms Version 1.1 - Draft 03. 2014.

26. Bromberg, Y.-D., P. Grace, and L. Réveillère. Starlink: runtime interoperability between heterogeneous middleware protocols. in Distributed Computing Systems (ICDCS), 201131 st International Conference on. 2011. IEEE. 